Editorial

\title{
Registration of clinical trials - a regional perspective
} M.F. Fathalla

\section{Scientific birth of the clinical trial in EMR}

The birth of the scientific clinical trial is often cited as an experiment by James Lind. On 20 May 1743, Lind compared the effect of a few supposed nutritional remedies against scurvy. The research subjects, 12 sailors suffering from scurvy on board the ship HMS Salisbury, were divided into 6 groups. In one group, each patient was given 2 oranges and 1 lemon every day, to eat upon an empty stomach. This was the group that showed marked improvement. Lind's experiment has sometimes been described as "the first deliberately planned therapeutic trial" [1]. However, centuries before Lind's experiment, a simple clinical trial was used by Abu Bakr Muhammad ibn Zakariyya al-Razi (865-925 CE), known to the Latin world as Rhazes in the 10th century $\mathrm{AD}$, to assess the value of bloodletting in cases of suspected meningitis [1]. The largest and most important of al-Razi's medical works is Kitab al-hawi fial-tibb (The comprehensive book of medicine), which was translated into Latin in the 13th century and repeatedly printed in Europe during the 15th and 16th centuries under the title Liber continens. Al-Razi's writings had a major influence on the development of medical practice in Europe [2]. In his writings, al-Razi's makes clear that he could not simply accept what he read, but had to investigate the effects of medicine himself before making a judgment. Al-Razi's approach to refining his treatment reflects a way of thinking about one of the principles of drawing inferences about the effects of treatments, namely, the need for comparison.

\section{What is a clinical trial?}

For the purposes of registration, a clinical trial is defined by the World Health Organization (WHO) [3] as "any study that prospectively assigns human participants or groups of humans to one or more health-related interventions to evaluate the effects on health outcomes." "Interventions include but are not restricted to drugs, cells and other biological products, surgical procedures, radiologic procedures, devices, behavioural treatments, process-of-care changes, preventive care, etc." This definition includes Phase I to Phase IV trials and has been adopted by the International Committee on Medical Journal Editors (ICMJE) [4].

\section{What is clinical trial registration?}

"WHO regards trial registration as the publication of an internationally agreed set of information about the design, conduct and administration of clinical trials. These details are published on a publicly-accessible website managed by a registry conforming to $\mathrm{WHO}$ standards" [3].

Twenty items of data are the minimum amount of information that must be disclosed at registration for each trial
$[3,4]$. These include a uniqueidentifying number, a statement of the intervention (or interventions) and comparison (or comparisons) studied, a statement of the study hypothesis, definitions of the primary and secondary outcome measures, eligibility criteria, key trial dates (registration date, anticipated or actual start date, anticipated or actual date of last follow-up, planned or actual date of closure to data entry, and date trial data are considered complete), target number of subjects, funding source and contact information for the principal investigator.

The registry must be accessible to the public at no charge. It must be open to all prospective registrants. It must be managed by a not-for-profit organization. There must be a mechanism to ensure the validity of the registration data. The registry should be electronically searchable.

\section{Why register clinical trials?}

The registration of all interventional trials is considered by WHO to be "a scientific, ethical and moral responsibility" [3].

\section{Enforcement of registration of clinical trials}

Registration of clinical trials is being enforced by one or more of the following mandates: legal mandate, ethical mandate, funding mandate and publication mandate.

A legal mandate allows governments to establish monitoring procedures and to penalize those who do not comply. "Registration of clinical trials is 
now a legal requirement in a number of countries" [5].

The ethical requirement is now mandated by the Declaration of Helsinki on Ethical Principles for Medical Research involving Human Subjects, 2008 Revision, which states that "Every clinical trial must be registered in a publicly accessible database before recruitment of the first subject". [6].

The funding mandate means that funding organizations and agencies can enforce registration by only financing trials that comply with the prospective registration requirement.

A publication mandate was laid down by the ICMJE when it decided that "The ICMJE member journals will require, as a condition of consideration for publication, registration in a public trials registry. Trials must register at or before the onset of patient enrolment. This policy applies to any clinical trial starting enrolment after July 1, 2005. For trials that began enrolment prior to this date, the ICMJE member journals will require registration by September 13,2005 , before considering the trial for publication" [7].

\section{Stakeholders in the registration of clinical trials}

Stakeholders in the registration of clinical trials include health care decisionmakers, trial participants, patients, ethics committees and institutional review boards, researchers, research managers, funding agencies and the public at large. They all stand to gain by prospective registration of clinical trials, and to lose if registration is not done.

Health care decision-makers need to ensure that decisions about health care are informed by ALL the available evidence. However, informed decisions cannot be properly made if publication bias and selective reporting are present. Regrettably there is selective reporting of clinical trials which leads to distortion of the evidence available for clinical decision-making. Trials that have negative findings (i.e. that show a new treatment is less effective than the standard treatment) or those that have no clear-cut findings are typically considered less interesting by researchers and journals alike. As organizations funding trials often have a vested interest in a certain outcome, trials that do not confirm that outcome and put financial interests at risk are particularly likely to remain unpublished [8].

\section{An essential role for WHO}

WHO has an essential role in the registration of clinical trial [9]. WHO's International Clinical Trial Registry Platform (ICTRP) provides access to information about ongoing and completed clinical trials. The platform links together "information held in national registers" and provides "a universal clinical trial number" [3]. An editorial in the British Medical Journal acknowledges the contribution of WHO stating that "Credit must also go to people within WHO for taking the lead in such an open and effective way. In doing so they have fulfilled two of WHO's core functions: the setting of norms and standards in medical research and health care, and working towards greater equity in health care." [8]

\section{Feasibility of establishing clinical trial registries in EMR}

In response to a resolution of the WHO Regional Committee of Ministers of Health in September 2011, the WHO Regional Office for the Eastern Mediterranean (EMRO) convened a workshop in Cairo from 31 October to 1 November 2011 to discuss the feasibility of establishing clinical trial registries conforming to WHO standards in countries of the Region and how EMRO could help in the process. The workshop brought together a high-level group of multi-disciplinary participants from 13 countries of the Region.

There was a consensus among the group of the importance of prospective registration of standard information for all clinical trials on a website accessible to all which conformed to WHO accepted standards. The need is urgent given the increasing numbers of clinical trials being conducted in the Region as noted in the country presentations, the emergence of contract research organization and the financial interests of the pharmaceutical industry.

Several valid questions were raised. Can we afford to do it if our resources are limited? Do we have the information technology infrastructure to support it? Do we have mechanisms to enforce it? A resource person from WHO Headquarters clarified, based on the experience in other countries, that the task was realistic and not very demanding in terms of financial and human resources. Moreover, several countries in the Region have already taken steps and action that can be built upon. The Islamic Republic of Iran, for example, has already established its national clinical trial registry, which has been accepted in the WHO Clinical Trial Registry Platform. Other countries (Jordan, Syrian Arab Republic and the United Arab Emirates) have already passed a law governing and regulating clinical trials. In Egypt, the Ministry of Health and Population is considering and planning such a law. There is already an electronically searchable clinical trial database in the American University in Beirut, planned to be accessible to the public. Registration of clinical trials is not an isolated activity, it is part of a wider spectrum of measures taken to ensure that society gets the complete benefit of this scientific endeavour. It is part of a chain, and in countries of the Region, many links in the chain are already in place.

Countries in the Region are diverse, and no one size fits all. The workshop recommended that EMRO tailors its 
technical support according to the capacity in the countries and the load of clinical trials conducted in each country. Some countries are to be encouraged to have their own national clinical trial registries, and EMRO and WHO Headquarters will provide the technical assistance to ensure that the registry conforms to WHO standards. For other countries, the workshop recommended that EMRO establish a regional clinical trial registry, which can act as intermediary between small clinical trial registries in countries and the WHO International Clinical Trial Registry Platform to ensure that that the data are accessible in an electronically searchable database.
For other countries that have not yet established their registries, the regional registry can be open to registrants. The over-riding concern, in whatever model is adopted, is to ensure that all clinical trials are prospectively registered in an electronically searchable and accessible database. This will make the information accessible to all in the Region, and to the world outside. The decisions on which model to adopt have to be made in the counties, and WHO should help them, as appropriate, to implement their decision.

The group made two comments worthy of note beyond the establishment of the registries. The first was the need to ensure the sustainability of the registry. The second was the need for the information provided on the database to be used for planning and decisions about directions of research in health. This will identify gaps in research, help to prevent wasteful duplication of research and identify opportunities for collaboration between researchers within and outside the Region working on the same issues.

The meeting ended on a positive and optimistic note, with one participant citing a Persian proverb: "You can if you want".

\section{References}

1. Hughes RE. James Lind and the cure of scurvy. An experimental approach. Medical History, 1975, 19:342-351.

2. Tibi S. Al-Razi and Islamic medicine in the 9th century. Journal of the Royal Society of Medicine, 2006, 99:206-207.

3. World Health Organization. International Clinical Trial Registry Platform (www.who.int/ictrp, accessed 3 January 2012).

4. Laine $\mathrm{C}$ et al. Clinical trial registration - looking back and moving ahead. Annals of Internal Medicine, 2007, 147:275-277.

5. Ghersi D, Pang T. From Mexico to Mali: four years in the history of clinical trial registration. Journal of Evidence-Based Medicine, 2009, 2(1):1-7.

6. World Medical Association. Declaration of Helsinki. Ethical principles for medical research involving human subjects.
Article 19. Adopted by the 18th WMA General Assembly, Helsinki, Finland, June 1964 and amended by the 59th WMA General Assembly, Seoul, October 2008 (www.wma.net/ en/30publications/10policies/b3/17c.pdf, accessed 3 January 2012)

7. De Angelis $\mathrm{C}$ et al. Clinical trial registration: a statement from the International Committee of Medical Journal Editors. Lancet, 2004, 364:911-912.

8. Godlee F. An international standard for disclosure of clinical trial information. British Medical Journal, 2006, 332:1107 [Erratum in: BMJ. 2006 Jun 17;332(7555):1418].

9. Evans T, Gukmezoglu M, Pang T. Registering clinical trials: An essential role for WHO. Lancet, 2004, 363:1413-1414. 